\title{
LIST OF MEMBERS AND GUESTS
}

Present at the Meeting of the Union at Paris, Fuly 10-17, I935

M. L. Zimmer

M. C. W. Allen

Dr W. E. Bernheimer

Dr S. Arend

Dr P. Bourgeois

Dr E. Delporte

Dr J. Delvosal

M. A. Dermul

M. F. De Roy

Dr F. Henroteau

Abbé G. Lemaître

M. L. Cap

Prof. N. Boneff

Prof. C. A. Chant

Dr J. S. Plaskett

M. Kao-Ping-Tsé

Dr Guth

M. Z. Kopal

Dr F. Link

Dr J. M. Mohr

Dr V. Nechvíle

M. C. Luplau-Janssen

Mme H. Nielsen

Prof. N. E. Nörlund

Dr M. R. Madwar
Argentine Republic.

Australia.

AUstria.

Dr K. Graff

BeLgIUM.

Prof. E. Merlin

Prof. F. Moreau

Général H. Seligmann

Prof. P. Stroobant

Dr P. Swings

Dr E. Vandekerkhove

Prof. H. L. Vanderlinden

M. J. Verbaandert

BRAzIL.

Bulgaria.

Canada.

Dr R. M. Stewart

China.

M. Pan-Puh

\section{Czecho-Slovakia.}

Mlle B. Nováková

Mlle S. Skelniková

M. Slouka

M. B. Sternberk

Prof. J. Svoboda

DenMaRK.

M. Strand

Prof. E. Strömgren

Mlle Vinter Hansen

EGYPT.

362 
France.

M. E. M. Antoniadi

M. Apoil

Mlle Bac

M. J. Baillaud

M. R. Baillaud

M. F. Baldet

M. Barbier

Général Bellot

M. Beloritzky

M. E. Belot

M. Bertaud

M. Bessemoulin

Mlle Bloch

M. Blum

M. Bonnet-Sainturier

Dr J. Bosler

Général Bourgeois

M. Brisse

M. A. Brun

Prof. H. Buisson

M. Camichel

Mlle Carravagia

M. Chalonge

Mme Chandon

M. A. Chatelu

M. J. Chatelu

Mlle Chaudun

Prof. J. Chazy

Prof. H. Chrétien

M. A. Claude

Mlle Clavier

M. Couder

M. A. Danjon

M. L. D'Azambuja

Comte A. de la Baume Pluvinel

M. de Granchamp

M. M. de Kerolyr

M. G. Delmotte

Dr H. Deslandres

M. L. Driencourt

M. Dubois-Chevalier

M. J. Dufay

M. L. Dunoyer

M. Durand

M. Ellsworth

Prof. E. Esclangon

Prof. C. Fabry

M. G. Fayet
M. E. Fichot

Mme A. Camille Flammarion

Mlle Fribourg

M. M. Giacobini

M. Godard

M. Goudey

M. H. Grouiller

M. R. Jarry-Desloges

Mlle Jasse

M. B. Jekhowsky

M. R. Jouaust

Mlle Kaufmann

M. I. Lagarde

M. Joanny Lagrula

M. J. Lagrula

M. C. Lallemand

M. A. Lambert

Mme Langier

Rev. P. P. Lejay, S.J.

M. B. Lyot

M. Maneng

M. Mantel

M. C. Maurain

M. Meyer

M. Michaud

M. H. Mineur

M. Montangerand

M. Nicolini

M. C. Nordmann

M. E. Paloque

M. A. Pérard

Général G. Perrier

M. E. Picard

M. Luc Picart

Amiral Pirot

M. F. Quénisset

Mme Isaac Roberts

M. Roger

M. G. Rougier

M. L. Rudaux

Dr P. Salet

M. Schaumasse

M. Schmitt

M. M. Simonin

M. H. Stoyko

M. Varchon

M. M. Wehrle

M. Ywanowitch 


\section{Great BRITAIN.}

Dr A. Beer

M. F. A. Bellamy

M. A. Bennett

Major Buckley

M. C. P. Butler

Prof. J. A. Carroll

Prof. S. Chapman

Dr L. J. Comrie

Dr A. C. D. Crommelin

M. C. R. Davidson

Prof. H. Dingle

Dr J. C. Dobbie

Sir Frank Dyson

Sir Arthur Eddington

M. J. Evershed

M. A. Fletcher

Prof. A. Fowler

M. W. M. H. Greaves

M. C. C. L. Gregory

Dr W. Hall

M. F. J. Hargreaves

M. W. T. Hay

M. H. P. Hollis

Dr C. V. Jackson

Dr J. Jackson

Capt. H. L. P. Jolly

Dr H. Spencer Jones

Dr H. Knox-Shaw
Major A. E. Levin

Capt. W. M. Lindley

Mlle F. Lowater

Prof. W. H. McCrea

M. T. L. Macdonald

M. J. C. P. Miller

Prof. E. A. Milne

Sir Robert Mond

M. A. M. Newbegin

M. H. W. Newton

M. J. W. Perry

Rev. T. E. R. Phillips

Prof. H. H. Plaskett

M. J. P. M. Prentice

Dr R. O. Redman

M. J. H. Reynolds

M. F. Robbins

Mlle Roberts

Rev. J. P. Rowland, S.J.

M. D. H. Sadler

Dr W. M. Smart

M. W. H. Steavenson

M. R. H. Stoy

Prof. F. J. M. Stratton

Dr R. Waterfield

M. E. G. Williams

Mlle $\mathbf{K}$. Williams

Brigadier H. St J. L. Winterbotham

\section{EAst INDIES (BRITISH).}

Dr S. Chandrasekhar

ITALY.

Prof. G. Abetti

Prof. G. Armellini

Prof. A. Bemporad

Prof. E. Bianchi

M. G. Cecchini

M. G. Horn d'Arturo

M. L. Gabba

M. L. Gratton
M. E. Loreta

Prof. M. Maggini

M. G. Righini

M. Sconze

Prof. G. Silva

Prof. L. Volta

M. F. Zagar

JAPAN.

Prof. K. Sotome

Dr A. Tanakadate 


\section{NetherLands.}

M. A. de Sitter

M. J. G. Ferwerda

Prof. E. Hertzsprung

Dr C. H. Hins

M. W. Martin

Dr M. Minnaert

Dr G. F. W. Mulders

Dr J. H. Oort

Prof. S. Rosseland

Prof. T. Banachiewicz

M. J. Gadomski

Dr M. Kamieński

Comte F. M. da Costa Lobo

M. G. S. da Costa Lobo

Prof. V. H. de Lemos

M. I. Armeanca

Prof. G. Bratu

Contre-Amiral C. Buchholzer

Prof. N. Coculesco

M. G. Demetrescu

M. N. Donitch

M. Dramba

Prof. P. Carrasco

M. R. Carrasco

Dr H. Castro

Prof. J. Comas y Sola

M. L. Herrero Garcia

Prof. O. Bergstrand

Prof. B. Lindblad

Prof. K. Lundmark

Prof. W. Brunner

Prof. E. Guyot
M. L. Plaut

M. W. J. A. Schouten

M. J. Uitterdijk

M. W. H. van den Bos

M. G. van Herk

M. J. A. Wesselink

Dr H. Zanstra

NORWAY.

POLAND.

Dr P. Kepiński

Dr J. Krassowski

Prof. J. Witkowski

Portugal.

M. M. dos Reis

M. J. C. Oom

M. M. Perez

ROUMANIA.

Lt. St Ioan

Col. V. Ioan

Dr C. Parvulesco

Prof. C. Popovici

Amiral Rizea

M. P. Sergesco

M. V. G. Siadbey

Spain.

M. E. Gastardi

Rev. L. Rodés, S.J.

M. P. M. Ryves

M. J. Tinoco

Dr J. M. Torroja

SwEDEN.

Dr N. Y. E. Nordenmark

M. Y. Ohman

Dr C. Schalén

\section{SwITZERLAND.}

Prof. T. Niethammer

Prof. G. Tiercy

365 
M. Okay

Dr W. S. Adams

Prof. B. Boss

Dr D. Brouwer

Prof. E. W. Brown

M. L. Campbell

Mlle A. Cannon

Dr K. Eckert

Prof. W. K. Green

M. Elmer Hall

Mlle Harwood

Capt. J. F. Hellweg

Prof. F. C. Jordan

M. Kovalenko

Dr J. Fessenkoff

Rev. J. J. A. Stein, S.J.

Prof. V. V. Michkovitch.

\section{TURKEY.}

\section{United States.}

M. W. D. Lambert

Prof. A. O. Leuschner

Rev. P. A. MacNally, S.J.

Dr W. F. Meggers

Prof. S. A. Mitchell

Dr J. Robertson

Prof. F. Schlesinger

Prof. H. Shapley

M. P. Sollenberger

Prof. F. Slocum

Dr P. van de Kamp

Dr A. van Maanen

\section{U.S.S.R.}

Prof. B. P. Gerasimovic

Vatican City.

\section{Yugosiavia.}

